科 学 通 报

\title{
振荡积分的快速算法 *
}

\author{
邓东香
}

(中山大学数学系, 广州 510275)

\section{关键词振荡积分、局部余弦基、稀疏矩阵}

振荡积分是指

$$
T(f)(x)=\int_{\mathbb{R}^{n}} \mathrm{e}^{i \pi p(x, y)} k(x-y) f(y) \mathrm{d} y,
$$

其中 $k(x)$ 是标准的 Calderon-Zygmund 核, 即 $k(x)=\Omega(x) /|x|^{n}, \Omega(x)$ 是 0 次齐次函数, 它在 $\Omega^{n}$ 的 单位球面上是足够光滑的, $p(x, y)$ 是任意的实值多项式. 近来年, 振荡积分 (1) 吸引了愈来愈 多的分析学家的注意. 关于它的 $L^{p}$ 有界性以及其他性质的研究, 可参看文献 $[1-4]$. 它的快 速算法还未被涉足过. 本文的目的是利用 Meyer 称之为时频小波的局部余弦基 (见文献[5]), 给出振荡积分 (1) 的快速算法. 实际上,我们要证的是:

定理 选择合适的局部余弦基, 振荡积分 (1) 在该基上的近似矩阵实现是稀疏的.

值得指出的是, 用局部余弦基, 得到声学散射中一类十分重要的振荡积分的快速算法, 是首先由 Bradie, Coifman 和 Grossman 作出的 ").

下面给出定理的证明.

\section{1 最简单的模型}

考虑振荡积分 (1) 的一个一维的最简单的情形

$$
T(f)(x)=\int_{-\infty}^{\infty} \mathrm{e}^{-i x x y} \frac{f(y)}{x-y} \mathrm{~d} y .
$$

给 因一个分划 $\mathbb{R}=\bigcup_{q=-\infty}^{\infty} I_{q}, I_{q}=[q, q+1]$. 选择光滑钟形函数 $b(x), \operatorname{supp} b(x) \subset\left[-\frac{1}{2}, \frac{3}{2}\right]$, 满足 $\sum_{q=-\infty}^{\infty} b_{q}^{2}(x)=1, b_{q}(x)=b_{q-1}(2 q-x)$, 其中 $b_{q}(x)=b(x-q)$. Coifman 和 Meyer 证明了 ${ }^{[0]}$ 全体

$$
C_{p}^{q}(x)=b(x-q) \cos \left[\left(p+\frac{1}{2}\right) \pi(x-q)\right],
$$

$q \in \mathbb{Z}, p \in \mathbb{Z}_{+}$, 组成 $L^{2}\left(\right.$ R) 的标准正交基. 它们是一种局部余弦基 $(L C B)$. 设 $L_{N}$ 是由基函数 $C_{p}^{q}(x)$, $-N \leqslant q \leqslant N, 0 \leqslant p \leqslant N$, 张成的 $L^{2}\left(\right.$ 圆) 的闭线性子空间. 我们实际上要实现的算子是 $T_{N}=P_{N} T P_{N}$, 其中 $P_{N}$ 是 $L^{2}$ (圆) 到 $L_{N}$ 的正交投影算子. 下面来估计 $T_{N}$ 在 $C_{p}^{q}(x)$ 的矩阵实现. 写出

1994-01-18 收稿.

*国家自然科学基金与中山大学高等学术研究中心基金资助项目。

1) Bradie, B., Coifman, R., Grossman, A., Preprint, Yale Univ.. 1993. 


$$
\begin{aligned}
\left(T C_{p}^{q}, C_{p^{\prime}}^{q^{\prime}}\right) & =\iint \frac{\mathrm{e}^{i \pi x y}}{x-y} b(y-q) \cos \left[\left(p+\frac{1}{2}\right) \pi(y-q)\right] b\left(x-q^{\prime}\right) \cos \left[\left(p^{\prime}+\frac{1}{2}\right) \pi\left(x-q^{\prime}\right)\right] \mathrm{d} y \mathrm{~d} x \\
& =\iint \frac{\mathrm{e}^{i n(u+q)(v+q)}}{(v-u)+\left(q^{\prime}-q\right)} b(u) b(v) \cos \left(p+\frac{1}{2}\right) \pi u \cos \left(p^{\prime}+\frac{1}{2}\right) \pi v \mathrm{~d} u \mathrm{~d} v .
\end{aligned}
$$

用指数函数表示余弦,化为估计下面的积分

其中

$$
A_{p p^{\prime}}^{q q^{\prime}}=\iint \varphi(u, v) \frac{\mathrm{e}^{\mathrm{in}\left[u\left(q^{\prime}+p\right)+v\left(q+p^{\prime}\right)\right]}}{(v-u)+\left(q^{\prime}-q\right)} \mathrm{d} u \mathrm{~d} v,
$$

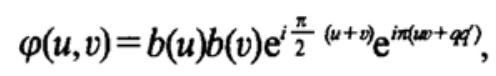

而 $-N \leqslant p, q, p^{\prime}, q^{\prime} \leqslant N$.

我们需要一个恒等式

$$
\iint_{\mathbb{R}^{2}} k(u-v) \varphi(u, v) \mathrm{e}^{i(u+l v)} \mathrm{d} u \mathrm{~d} v=\int_{-\infty}^{\infty} \hat{k}(\xi) \hat{\varphi}(k-\xi, l+\xi) \mathrm{d} \xi,
$$

其中 $\hat{k}, \hat{\varphi}$ 分别表示 $k$ 与 $\varphi$ 的一元与二元的 Fourier 变换. (3) 式的证明并不难, 可参阅脚注 1). 用公式 (3), 便有

$$
A_{p f^{\prime}}^{q q^{\prime}}=\int_{-\infty}^{\infty} \hat{\varphi}\left(q^{\prime}+p-\xi, q+p^{\prime}+\xi\right) \mathrm{e}^{i\left(q^{\prime}-q\right) \xi} \operatorname{sgn} \xi \mathrm{d} \xi
$$

取 $\theta_{1}(\xi), \theta_{2}(\xi) \in C^{\infty}$, 满足 $\theta_{1}(\xi)=1$ 当 $|\xi| \leqslant \frac{1}{2} . \operatorname{supp} \theta_{1} \subset[-1,1]$, 且 $\theta_{1}(\xi)+\theta_{2}(\xi)=1$. 这样 $A_{p p^{\prime}}^{q q^{\prime}}=I_{1}+I_{2}$, 其中

$$
I_{j}=\int \hat{\varphi}\left(q^{\prime}+p-\xi, q+p^{\prime}+\xi\right) \theta_{j}(\xi) \mathrm{e}^{i\left(q^{\prime}-q\right)} x_{\operatorname{sgn}}^{*} \xi \mathrm{d} \xi, \quad j=1,2 .
$$

由于 $\varphi$ 支于 $|u| \leqslant 2,|v| \leqslant 2$, 且任意阶光滑, 有

对任意固定的 $m>0$ 成立. 因此

$$
|\hat{\varphi}(u, v)| \leqslant C /(1+|u|+|v|)^{m},
$$

类似地

$$
\left|I_{1}\right| \leqslant C /\left(1+\left|q^{\prime}+p\right|+\left|q+p^{\prime}\right|\right)^{m}
$$

$$
\begin{aligned}
\left|I_{2}\right| & \leqslant \int_{|q|>1 / 2}\left|\hat{\varphi}\left(q^{\prime}+p-\xi, q+p^{\prime}+\xi\right)\right| \mathrm{d} \xi \leqslant \int_{|\mu| \geqslant c / 2}\left|\hat{\varphi}\left(q^{\prime}+p-q-p^{\prime}-2 u, q+p^{\prime}+q^{\prime}+p\right)\right| \mathrm{d} u \\
& \leqslant C /\left(1+\left|q+p^{\prime}+q^{\prime}+p\right|\right)^{m} .
\end{aligned}
$$

另外, 容易看出

由此便得

$$
\sum_{|\alpha|<m} \int_{-\infty}^{\infty}\left|\frac{\mathrm{d}^{\alpha}}{\mathrm{d} \xi^{\alpha}} \hat{\varphi}\left(q^{\prime}+p-\xi, q+p^{\prime}+\xi\right)\right| \mathrm{d} \xi \leqslant C 2^{m},
$$

1) 同 2123 页脚注. 


$$
\left|I_{2}\right| \leqslant C /\left(1+\left|\frac{q^{\prime}-q}{2}\right|\right)^{m}
$$

总起来, 便得到

$$
A_{p^{\prime}}^{q^{\prime}}=O\left(\frac{1}{\left(1+\left|q^{\prime}+p\right|+\left|q+p^{\prime}\right|\right)^{m}}\right)+O\left(\frac{1}{\left(1+\left|\frac{q^{\prime}-q}{2}\right|+\left|q^{\prime}+p+q+p^{\prime}\right|\right)^{m}}\right) .
$$

故对任意 $\varepsilon>0$, 存在常数 $B$, 使得 $\left|A_{p p^{\prime}}^{q q^{\prime}}\right|>\varepsilon$ 蕴含了

$$
\left|p+q^{\prime}\right|<B, \quad\left|q+p^{\prime}\right|<B,
$$

或者

$$
\left|q^{\prime}-q\right|<2 B,\left|q^{\prime}+p+q+p^{\prime}\right|<B .
$$

这表明, 在 $4 N^{2} \times 4 N^{2}$ 矩阵 $\left\{A_{p \phi}^{q \alpha}\right\}$ 中, 满足 $\left|A_{\rho f}^{q f}\right|>\varepsilon$ 的元素数目, 不超过 $C B^{2} N^{2}$. 也就是说, 矩阵 $\left\{A_{p p^{\prime}}^{q p^{\prime}}\right\}$ 是稀疏的.

\section{2 位相为一般实多项式的情形}

为把上节的结果推广到位相为一般实多项式的情形, 我们只需考虑

$$
T(f)(x)=\int_{-\infty}^{\infty} \mathrm{e}^{\mathrm{inx} x^{2} y} \frac{f(y)}{x-y} \mathrm{~d} y .
$$

读者可以从下面的推理发现, 只需对推理稍加修改, 便可用于位相为一般的实多项式的情 形.

对任意固定的 $N$, 我们用 $I_{q}=\left(\frac{q}{\sqrt{N}}, \frac{q+1}{\sqrt{N}}\right), q \in \mathbb{Z}$, 给出 圆的一个分划. 这样, 全体

$$
C_{p}^{q}(x)=N^{1 / 4} b(\sqrt{N} x-q) \cos \left[\left(p+\frac{1}{2}\right) \pi(\sqrt{N} x-q)\right],
$$

$q \in \mathbb{Z}, p \in \mathbb{Z}_{+}$, 组成 $L^{2}($ 圆 $)$ 的标准正交基. 定义 $L_{N}$ 为由基函数 $C_{p}^{q}(x),-N^{3 / 2} \leqslant q \leqslant N^{3 / 2}, 0 \leqslant p \leqslant N^{3 / 2}$,

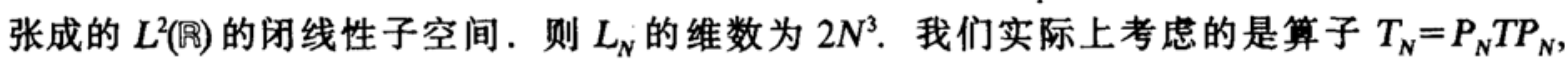
其中 $P_{N}$ 是 $L^{2}$ (圆) 到 $L_{N}$ 的正交投影算子.

用类似于上节的推理, 我们要估计的是

$$
\left.A_{p p^{\prime}}^{q q^{\prime}}=\iint \frac{b(u) b(v) \mathrm{e}^{i \frac{\pi}{2}(u+v)}}{(v-u)+\left(q^{\prime}-q\right)} \mathrm{e}^{i n\left[\left(\frac{u+q}{\sqrt{N}}\right)\left(\frac{v+q}{\sqrt{N}}\right)^{2}+p u+p^{\prime} v\right.}\right] \mathrm{d} u \mathrm{~d} v,
$$

其中 $-N^{3 / 2} \leqslant p, p^{\prime}, q, q^{\prime} \leqslant N^{3 / 2}$. 用公式 (3), 便有

其中

$$
A_{p p^{\prime}}^{q q^{\prime}}=\int_{-\infty}^{\infty} \hat{\varphi}\left(N^{3 / 2}\left(\bar{q}^{\prime 2}+\bar{p}\right)-\xi, N^{3 / 2}\left(2 \overline{q q^{\prime}}+\overline{p^{\prime 2}}\right)+\xi\right) \mathrm{e}^{i\left(q^{\prime}-q\right)} \operatorname{sgn} \xi \mathrm{d} \xi
$$

$$
\left.\varphi(u, v)=b(u) b(v) \mathrm{e}^{i \frac{\pi}{2}(u+v)} \mathrm{e}^{i\left[\frac{u^{2}}{N^{3 / 2}}+2 \overline{q^{\prime}} u v+\bar{q} v^{2}+N^{3} \bar{q}^{3}-\bar{q}\right.}\right], \quad \bar{p}=p / N^{3 / 2}, \quad \bar{q}=q / N^{3 / 2}, \quad \overline{p^{\prime}}=p^{\prime} / N^{3 / 2}, \quad \overline{q^{\prime}}=q^{\prime} / N^{3 / 2} .
$$

而且 $-1 \leqslant \bar{p}, \bar{q}, \bar{p}^{\prime}, \bar{q}^{\prime} \leqslant 1$. 用类似于上节的方法, 可估计出 


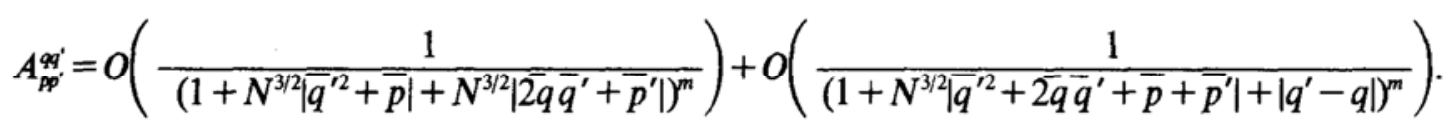

因此, 对任意给定的 $\varepsilon>0$, 存在常数 $B$, 使得 $\left|A_{p p^{\prime}}^{q q^{\prime}}\right|>\varepsilon$ 蕴含了

或者

$$
\left|p+q^{2} / N^{3 / 2}\right|<B, \quad\left|p^{\prime}+2 q q^{\prime} / N^{3 / 2}\right|<B,
$$

$$
\left|q^{\prime}-q\right|<B, \quad\left|p+p^{\prime}+2 q q^{\prime} / N^{3 / 2}+q^{\prime 2} / N^{3 / 2}\right|<B .
$$

这表明, 在 $4 N^{3} \times 4 N^{3}$ 矩阵 $\left\{A_{p p^{\prime}}^{q q^{\prime}}\right\}$ 中, 满足 $\left|A_{p p^{\prime}}^{q q^{\prime}}\right|>\varepsilon$ 的元素数目, 不超过 $C B^{2} N^{3}$. 也就是说, 矩阵 $\left\{A_{p p^{\prime}}^{q p^{\prime}}\right\}$ 是稀疏的.

\section{3 推广到高维的情形}

从上面的推理, 不难看出, 只需要用高维的局部三角函数基, 便可对高维的振荡积分 (1)证 明定理. 因此,定理证毕.

上面的推理还表明, 我们的定理,对下面的振荡积分

$$
T(f)(x)=\int_{\mathbb{R}^{n}} \mathrm{e}^{i \varphi(x, y)} k(x-y) f(y) \mathrm{d} y,
$$

仍然成立, 只要 $\varphi(x, y)$ 是光滑的实值函数, 其各阶导数一致有界.

致谢 本文是作者在访问 Yale 大学期间完成的. 作者对 R. Coifman 教授对有关问题的 讨论与建议,表示深切的感谢.

\section{参考 文献}

[1] Rioci, F., Stein, E., Proc. Natl. Acad. USA, 1986, 83:1-3.

[2] Ricci, F., Stein, E., J. of Funct. Anal., 1987, 73: 178-184.

[3] Stein, E., Ann. Math. Stud., Princeton University Press, 1986, 112:307-355.

[4] Stein, E., Harmonic Analysis. Real-Variable Method, Orthogonality and Oscillatory Integrals, Princeton University Press, 1993.

[5] Meyer, Y., Wavelets: Algorithms and Applications, SIAM, 1993.

[6] Coifman, R., Meyer, Y., C. R. Acad. Sci. Paris, D, 1991, 312, Ser. I, 259-261. 